\title{
A mini-review of the validity, quality and efficacy of candidate vaccines in controlling the COVID-19
}

\author{
Hedyeh Maghareh Abed ${ }^{1}$, Pardis Piri Dizaji², Hamed Hekmatnezhad3, Hoda Sabati4, Donya Zare5,* \\ ${ }^{1}$ Department of Biochemistry, Faculty of Advanced Science and Technology, Tehran Medical Science, Islamic Azad University, Tehran, \\ Iran \\ 2Department of Biology, Faculty of Basic Sciences, Azarbaïan Shahid Madani University, Tabriz, Iran \\ 3Department of Basic Sciences, Sari Agricultural Sciences and Natural Resources University, Sari, Iran \\ 4Biotechnology and Biological Science Research Center, Faculty of Science, Shahid Chamran University of Ahvaz, Iran \\ 5Department of Microbiology, School of Medicine, Isfahan University of Medical Sciences, Isfahan, Iran
}

\begin{abstract}
Few would have thought that in this century, a new coronavirus called SARS-CoV-2 would kill many people around the world, cripple the economy, and leave the medical staff helpless. Severe acute respiratory syndrome coronavirus 2 (SARS-CoV-2), a virus that first appeared in Wuhan, China, and spread rapidly around the world, and strict quarantines did not prevent the severe prevalence from spreading worldwide. Antiviral drugs do not work well enough for everyone. The mortality rate in the world is still significant. The only thing that gives hope to the people of the world is the hope of being vaccinated, so by producing vaccines in the shortest possible time, science has once again saved humanity. Thus, from the very beginning, pharmaceutical companies started to produce safe vaccines. Currently, more than 200 types of vaccines around the world are undergoing various stages of production, and about 30 vaccines have entered the clinical trial phase, of which 9 vaccines have entered phase 1 to 3 of clinical trials. DNA and RNA-based vaccines were first developed and were not licensed before coronavirus disease 2019 (COVID-19). Other types of vaccines, including nonreplicating viral vectors as well as inactivated vaccines, are undergoing clinical phases. There are currently 9 common vaccines Inovio Pharmaceuticals, Moderna, BioNTech/Pfizer, AstraZeneca, CanSino Biological, Gam-COVID-Vac (Sputnik V), Wuhan Institute of Biological Products/Sinopharm, Beijing Institute of Biological Products/Sinopharm, and Sinovac Institutes in the world. Vaccination with the Pfizer vaccine, which is approved by the World Health Organization (WHO), is underway in many countries. The WHO predicts that by the end of 2021, one billion people worldwide will be vaccinated by the company.
\end{abstract}

Keywords: SARS-CoV-2, COVID-19, Vaccine, Pfizer, Moderna, AstraZeneca

\section{Introduction}

Almost one year has passed since the emergence of the deadly coronavirus. The virus was first identified in 2019 in Wuhan, China. The virus has spread rapidly around the world, killing many people every day $[1,2]$. According to official sources, 106 million people have been infected with the virus, of which 59 million have been identified and about 2 million have died.

\footnotetext{
${ }^{*}$ Corresponding author:

Donya Zare, MSc

Department of Microbiology, School of Medicine,

Isfahan University of Medical Sciences, Isfahan, Iran

Tel/Fax: +98912 7560121

Email: d.zare96@gmail.com

https://orcid.org/o0oo-0001-7827-5600
}

Received: January, 21, 2021

Accepted: February, 09, 2021
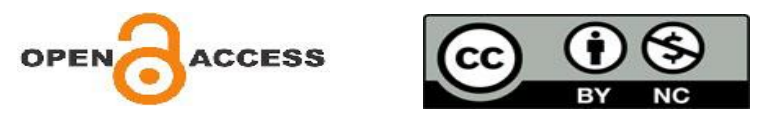
Coronavirus disease 2019 (COVID-19) disease which is caused by severe acute respiratory syndrome coronavirus-2 (SARS-CoV-2), covers a wide range of clinical symptoms, from mild symptoms such as mild flu to severe symptoms such as acute respiratory distress syndrome (ARDS) followed by pneumonia and death. It should be noted that flock safety is possible by gaining natural immunity through infection, but the losses and consequences can be irreparable [3]. Swedish authorities believed that with $60 \%$ of the population affected, herd immunity could be effective in controlling the disease, a hypothesis that was ineffective and that the death rate in Sweden was five times higher than in Germany [4]. Thus, the production of an effective vaccine against the disease was prioritized and is considered as the only practical way to establish herd safety. The incubation period of COVID-19 is estimated to be 5 days and the onset of symptoms at 11.5 days [5]. Studies show that patients with COVID-19 excrete the highest levels of viral nucleic acid (RNA) at the onset of symptoms [6]. These and other epidemiological data suggest disease transmission in a pre-symptom period [7]. It takes two weeks for the severe symptoms of the disease to appear followed by a cytokine storm created by immunity which causes widespread inflammation and diffuse intravascular coagulation $[8,9]$. Studies showed the mortality rate of this disease in patients is age-related. A high percentage of people over the age of seventy who have an underlying disease is dying. Other factors such as high blood pressure, obesity, diabetes as well as gender (male $>$ female) affect the severity of the disease [10-12]. Some anti-CoV candidate drugs should be analyzed as preclinical compounds for the potential treatment of COVID-19. A small number of COVID-19 candidate drugs have reached phase 3 clinical trials such as ritonavir, cobicistat, lopinavir, darunavir, remdesivir, HIV protease inhibitor (ASCo9F), and oseltamivir [13]. Inhibitors of the SARS-CoV or Middle East respiratory syndrome (MERS) that were previously available, are being investigated [14]. Many institutions around the world have been working from the beginning to develop an effective vaccine against COVID-19. In August 2020, more than 200 candidate vaccines were introduced at different stages of development. While about 30 vaccines are currently in clinical trials, the 9 vaccines that have entered phase 1 clinical trials since mid-May 2020 will include AstraZeneca/Oxford's AZD1222 and
Moderna's mRNA-1273 vaccines. mRNA-1273 in the Moderna vaccine encodes the $S$ protein [15]. S protein is released, which stimulates cellular and humoral immune responses. Earlier, RNA interference technique was used in the treatment of SARS and MERS, but it did not reach the vaccine production stage [16]. mRNA vaccines are a suitable alternative to existing vaccines due to their high efficacy, appropriate immune response, high production rate, harmless administration, and easy processing [17]. In general, vaccines work by training the immune system to identify the part of the virus that causes the disease. In the traditional method, the inactivated virus was used to make the vaccine, but in the case of the mRNA vaccine, the situation is different because instead of injecting the viral protein, the person receives the genetic material (mRNA) that encodes the viral protein. When these genetic instructions are injected into the arms, the muscle cells translate these codes to make viral proteins directly in the body. The mRNA vaccine encodes only an important fragment of a viral protein. mRNAs show the immune system what the real virus will cause in the body, and this preview will allow the immune system to design powerful antibodies and elicit an appropriate immune response. mRNA is not transmitted to the next generation [18]. Given the massive COVID-19 pandemic, production capacity soon will certainly not be able to meet global demand for the vaccine in all countries, so vaccination is a priority for medical staff and susceptible individuals. It is estimated that by the end of 2021, the vaccine will be available worldwide to the rest of the population. Accuracy and sensitivity in performing preclinical and clinical trials in order to produce a safe and effective vaccine is of great importance in order to prevent severe side effects in individuals [19]. The process of producing a new vaccine is long and usually takes 10 to 15 years. Before to COVID-19, the mumps vaccine was the fastest vaccine produced and used in 5 years. Thus, producing a vaccine against COVID-19 over a period of one to two years is a challenge that researchers have finally been able to accomplish. The exploratory phase is the first step in the vaccine production process, which involves preliminary laboratory research that involves identifying the structure and genetics of the virus. The second phase is a pre-clinical studies in which experiments are performed on an animal model such as a mouse to evaluate the immune response of the 
candidate vaccine and its advantages and disadvantages. Clinical trials are performed on humans after the safety and efficacy of the vaccine have been demonstrated in animal models. Clinical trials in humans are performed in three phases. In phase 1 (Safety), the vaccine is injected into a small number of healthy and immunocompetent individuals. In phase two (Expanded Safety), the vaccine is tested on hundreds of people in different groups. At this stage, should be evaluated the immune response, appropriate dose, and distance between doses. Phase 2 confirms the safety of the vaccine and you can also determine the appropriate dose to test in phase 3. In Phase 3 (Efficacy) clinical trials in humans, the vaccine is tested on a large scale in thousands of people. In this step, will be evaluated the effect of the vaccine [20]. There are various platforms being looked at for the development of COVID-19 vaccines. These include RNA, DNA, nonreplicating viral vectors and inactivated vaccines.

\section{RNA-based vaccines}

The RNA-based platform, developed by Moderna and Pfizer/BioNTech companies, is a candidate in clinical trials and is in phase 3 clinical trials. The type of candidate vaccine for both Moderna and Pfizer is lipid nanoparticle [LNP] encapsulated mRNA. The target antigen in both of them is spike protein, as well as multiple doses. They will also trigger both humoral and cellular immune responses. Their advantage is that they are made using genetic sequencing and do not need to be cell-cultured. One of their disadvantages is that LNP is temperature-sensitive, and no RNA-based vaccine has been developed so far. Currently, both vaccines are approved by the US government [21, 22].

\section{DNA-based vaccines}

DNA-based vaccines encode the target antigen and produce a persistent immune response. Transfected cells continuously produce certain transgenic proteins. DNA vaccines are temperature stable, but their safety and efficacy require further research. Administration of DNA vaccines may also cause mutations and integration of the host gene [23]. The DNA-based platform, developed by Inovio Pharmaceuticals (an American biotechnology company), is a candidate in clinical trials and is in phases 1 and 2 clinical trials. The type of candidate vaccine for Inovio Pharmaceuticals is DNA plasmid vaccine with electroporation. The vaccine, called INO4800 , is injected intradermally followed by electroporation to ensure that it is absorbed into the cell. The target antigen in these vaccines, such as RNAbased vaccines, is spike protein, as well as multiple doses. They will also trigger both humoral and cellular immune responses. The most important advantage of this type of vaccine is that electroporation produces a strong immune response and does not require cell culture. One of the disadvantages of the DNA-based vaccine is that electroporation, although safe, can potentially be problematic, and no DNA-based vaccine has been developed in the past [24, 25].

\section{Non-replicating viral vector vaccines}

The Non-replicating Viral Vector platform, developed by the AstraZeneca/University of Oxford and CanSino Biological Inc/Beijing Institute of Biotechnology, and both are in phase 3 and phase 2 clinical trials, respectively. The type of candidate vaccine for AstraZeneca is AZD1222 and for CanSino is Adenovirus type 5 vector. Both AstraZeneca and CanSino utilize adenovirus as a vector for their COVID-19 vaccine. The target antigen in both vaccines is spike protein, and the AstraZeneca vaccine is a single dose. They will also trigger both humoral and cellular immune responses. The advantage of the nonreplicating viral vector vaccine of AstraZeneca is that it can be produced on a large scale and provides an effective immune response, as previously demonstrated in the case of Ebola. One of the disadvantages of the AstraZeneca vaccine from the University of Oxford is that the availableimmunity can disrupt clinical use and reduce the immune response. CanSino and AstraZeneca intend to offer their vaccines at a low cost, which, given their moderate effectiveness, may be beneficial to some countries [2628]. Gam-COVID-Vac (Sputnik V) from Russia is a heterologous recombinant adenovirus (rAd)-based vaccine. The immunity of the vaccine is satisfactory and produces a strong humoral and cellular immune response and its efficacy is 92\% [29].

\section{Inactivated vaccines}

The Inactivated platform, developed by Wuhan Institute of Biological Products/Sinopharm, Beijing Institute of Biological Products/Sinopharm, and Sinovac Institutes. They are all in phase 3 clinical 
trials. The type of candidate vaccine for Wuhan Institute is inactivated and for Sinovac Institutes is inactivated aluminum adjuvant. The target antigen in the vaccine produced by these three institutes is the whole virus, and the only Wuhan Institute is the multiple-dose. The immune response created by the Wuhan Institute vaccine is more humoral, and also the immune response at the Sinovac Institute is mostly humoral, which will increase in the presence of aluminum adjuvant. The advantage of using the Wuhan Institute inactived vaccine is that, due to the use of the inactive virus, the pathogen is killed and there is no risk of recurrence [30, 31].

\section{Conclusion}

In this short review, we discuss ways that can prevent the deadly spread of COVID-19 worldwide, including drug therapy and vaccine use. Eventually, the efforts of researchers around the world led to the development of effective vaccines against COVID-19 to be the sweet end to the most terrible nightmare of the 21st century. Of course, vaccination should be done by adopting policies not only in rich countries, but in all countries regardless of nationality, religion, color, and race, and governments should do their best for the health of the people. Predictably, the only way to control COVID-19 before vaccination by countries is to use new antiviral drugs, social distancing, using facemasks, and regular handwashing with soap and water and if soap and water are not readily available and hands are not visibly dirty, use a hand sanitizer that contains at least $60 \%$ alcohol. Currently, the BNT162b2 vaccine from Pfizer/BioNTech pharmaceutical companies with $90 \%$ efficiency and mRNA-1273 vaccine from Moderna company has been approved by the US government and is being vaccinated with the Pfizer vaccine in the United States, many European countries, and several Asian countries [21]. The most important challenge for mRNA vaccine production is its inherent instability, as it is more likely to degrade above freezing temperature. Pfizer/BioNTech mRNA vaccines require optimal storage at minus 94 Fahrenheit degree and are destroyed in about five days at room temperature slightly above freezing. In contrast, Moderna Pharmaceuticals claims that their vaccine can be stored at most home temperatures or medical freezers for up to six months for long-term transportation and storage. The AstraZeneca vaccine, despite being less effective (70\%) than its competitors such as Pfizer and Moderna, can be used in standard refrigerated conditions between 36 and 46 Fahrenheit degree for at least six months for storage and transportation, which is a valuable advantage over two The vaccine rivals Pfizer and Moderna and will be easier to use in developing countries [22]. Pharmaceutical companies Pfizer and Moderna have not joined the COVAX initiative, but AstraZeneca has agreed to provide lower-cost vaccines to middle-income countries. For all COVID-19 vaccine candidates, we have only preliminary information on a small number of infections, and none of the COVID-19 vaccineproducing groups have released complete information so far, so it is difficult to fully assess the differences between them. However, for evaluating the effectiveness of all COVID-19 vaccines to control the pandemic of this disease, we must wait for more follow-up and long-term data.

\section{Author Contributions}

All authors contributed equally, and read and approved the final version of manuscript.

\section{Conflict of Interest}

We declare that we have no conflict of interest.

\section{Ethical declarations}

Not applicable.

\section{Financial Support}

\section{None.}

\section{References}

1. Ahn DG, Shin HJ, Kim MH, Lee S, Kim HS, Myoung J, et al. Current Status of Epidemiology, Diagnosis, Therapeutics, and Vaccines for Novel Coronavirus Disease 2019 (COVID-19). J Microbiol Biotechnol. 2020; 30(3):313-24.

2. Merad M, Martin JC. Pathological inflammation in patients with COVID-19: a key role for monocytes and macrophages. Nat Rev Immunol. 2020; 20(6):355-62.

3. Randolph HE, Barreiro LB. Herd Immunity: Understanding COVID-19. Immunity. 2020; 52(5):737-41.

4. Jung F, Krieger V, Hufert FT, Küpper JH. Herd immunity or suppression strategy to combat COVID-19. Clin Hemorheol Microcirc. 2020; 75(1):13-7.

5. Lauer SA, Grantz KH, Bi Q, Jones FK, Zheng Q, Meredith HR, et al. The Incubation Period of Coronavirus Disease 2019 (COVID19) From Publicly Reported Confirmed Cases: Estimation and Application. Ann Intern Med. 2020; 172(9):577-82. 
6. Zou L, Ruan F, Huang M, Liang L, Huang H, Hong Z, et al. SARS-CoV-2 Viral Load in Upper Respiratory Specimens of Infected Patients. N Engl J Med. 2020; 382(12):1177-9.

7. He X, Lau EHY, Wu P, Deng X, Wang J, Hao X, et al. Temporal dynamics in viral shedding and transmissibility of COVID-19. Nat Med. 2020; 26(5):672-5.

8. Zhou F, Yu T, Du R, Fan G, Liu Y, Liu Z, et al. Clinical course and risk factors for mortality of adult inpatients with COVID-19 in Wuhan, China: a retrospective cohort study. Lancet. 2020; 395(10229):1054-62.

9. Tang N, Li D, Wang X, Sun Z. Abnormal coagulation parameters are associated with poor prognosis in patients with novel coronavirus pneumonia. J Thromb Haemost. 2020; 18(4):844-7. 10. Bhatraju PK, Ghassemieh BJ, Nichols M, Kim R, Jerome KR, Nalla AK, et al. Covid-19 in Critically Ill Patients in the Seattle Region - Case Series. N Engl J Med. 2020; 382(21):2012-22.

11. Huang C, Wang Y, Li X, Ren L, Zhao J, Hu Y, et al. Clinical features of patients infected with 2019 novel coronavirus in Wuhan, China. Lancet. 2020; 395(10223):497-506.

12. Guan WJ, Liang WH, Zhao Y, Liang HR, Chen ZS, Li YM, et al. Comorbidity and its impact on 1590 patients with COVID-19 in China: a nationwide analysis. Eur Respir J. 2020; 55(5):2000547. 13. Li G, De Clercq E. Therapeutic options for the 2019 novel coronavirus (2019-nCoV). Nat Rev Drug Discov. 2020; 19(3):14950 .

14. Dhama K, Sharun K, Tiwari R, Dadar M, Malik YS, Singh KP, et al. COVID-19, an emerging coronavirus infection: advances and prospects in designing and developing vaccines, immunotherapeutics, and therapeutics. Hum Vaccin Immunother. 2020; 16(6):1232-8.

15. Zhang J, Zeng H, Gu J, Li H, Zheng L, Zou Q. Progress and Prospects on Vaccine Development against SARS-CoV-2. Vaccines (Basel). 2020; 8(2):153.

16. Ahmadi A, Hekmatnezhad H. The sound of getting rid of coronavirus by RNA interference technology: RNAi against COVID-19. J Curr Biomed Rep. 2020; 1(2):45-7.

17. Wang F, Kream RM, Stefano GB. An Evidence Based Perspective on mRNA-SARS-CoV-2 Vaccine Development. Med Sci Monit. 2020; 26:e924700.

18. JahanafroozZ, Baradaran B, Mosafer J, Hashemzaei M, Rezaei T, Mokhtarzadeh A, et al. Comparison of DNA and mRNA vaccines against cancer. Drug Discov Today. 2020; 25(3):552-60.

19. Sempowski GD, Saunders KO, Acharya P, Wiehe KJ, Haynes BF. Pandemic Preparedness: Developing Vaccines and Therapeutic Antibodies For COVID-19. Cell. 2020; 181(7):145863.

20. Han S. Clinical vaccine development. Clin Exp Vaccine Res. 2015; 4(1):46-53.
21. Triggle CR, Bansal D, Farag EABA, Ding H, Sultan AA. COVID19: Learning from Lessons To Guide Treatment and Prevention Interventions. mSphere. 2020; 5(3):e00317-20.

22. Mulligan MJ, Lyke KE, Kitchin N, Absalon J, Gurtman A, Lockhart S, et al. Phase I/II study of COVID-19 RNA vaccine BNT162b1 in adults. Nature. 2020; 586(7830):589-93.

23. Belete TM. Review on Up-to-Date Status of Candidate Vaccines for COVID-19 Disease. Infect Drug Resist. 2021; 14:151-61.

24. Tebas P, Yang S, Boyer JD, Reuschel EL, Patel A, ChristensenQuick A, et al. Safety and immunogenicity of INO-4800 DNA vaccine against SARS-CoV-2: A preliminary report of an openlabel, Phase 1 clinical trial. EClinicalMedicine. 2021; 31:100689.

25. INOVIO Announces Positive Interim Phase 1 Data for INO4800 Vaccine for COVID-19. Retrieved January 02, 2021. Available at: http://ir.inovio.com/news-releases/news-releasesdetails/2020/INOVIO-Announces-Positive-Interim-Phase-1Data-For-INO-480o-Vaccine-for-COVID-19/default.aspx. 26. Ramasamy MN, Minassian AM, Ewer KJ, Flaxman AL, Folegatti PM, Owens DR, et al. Safety and immunogenicity of ChAdOx1 nCoV-19 vaccine administered in a prime-boost regimen in young and old adults (COVoO2): a single-blind, randomised, controlled, phase 2/3 trial. Lancet. 2021; 396(10267):1979-93. 27. Zhu FC, Li YH, Guan XH, Hou LH, Wang WJ, Li JX, et al. Safety, tolerability, and immunogenicity of a recombinant adenovirus type- 5 vectored COVID- 19 vaccine: a dose-escalation, open-label, non-randomised, first-in-human trial. Lancet. 2020; 395(10240):1845-54.

28. Sumner M. Why One Vaccine is Already a Sure Loser, and also a Sure Winner, in the Race to Inoculate the World. Retrieved December 25, 2020. Available at: https://www.dailykos.com/stories/2020/7/24/1963291/-Whyone-vaccine-is-already-a-sure-loser-and-also-a-sure-winner-inthe-race-to-inoculate-the-world.

29. Logunov DY, Dolzhikova IV, Shcheblyakov DV, Tukhvatulin AI, Zubkova OV, Dzharullaeva AS, et al. Safety and efficacy of an rAd26 and rAd5 vector-based heterologous prime-boost COVID19 vaccine: an interim analysis of a randomised controlled phase 3 trial in Russia. Lancet. 2021. [In press]. doi: 10.1016/So1406736(21)00234-8.

30. Xia S, Duan K, Zhang Y, Zhao D, Zhang H, Xie Z, et al. Effect of an Inactivated Vaccine Against SARS-CoV-2 on Safety and Immunogenicity Outcomes: Interim Analysis of 2 Randomized Clinical Trials. Jama. 2020; 324(10):951-60.

31. Wang H, Zhang Y, Huang B, Deng W, Quan Y, Wang W, et al. Development of an Inactivated Vaccine Candidate, BBIBP-CorV, with Potent Protection against SARS-CoV-2. Cell. 2020; 182(3):713-21.e9. 